\title{
Research Paper \\ The Effectiveness of Mindfulness-Based Stress Reduction on the Depression, Anxi- ety, Stress, and Pain Perception in Females with Obstructed Labour-Induced Chronic Low Back Pain
}

\author{
Elaheh Sobhani ${ }^{1} \oplus{ }^{*}{ }^{*}$ Narges Babakhani ${ }^{1} \oplus$, Mahmoud Reza Alebouyeh ${ }^{3} \mathbb{C}$
}

1. MA. in Psychology, Department of Psychology, Faculty of Psychology and Social Sciences, Roodehen Branch, Islamic Azad University, Roodehen, Iran. 2. PhD. in Psychology, Department of Psychology, Faculty of Psychology and Social Sciences, Roodehen Branch, Islamic Azad University, Roodehen, Iran 3. Associate Professor, Department of Anesthesiology, School of Medicine, Iran University of Medical Sciences, Tehran, Iran.

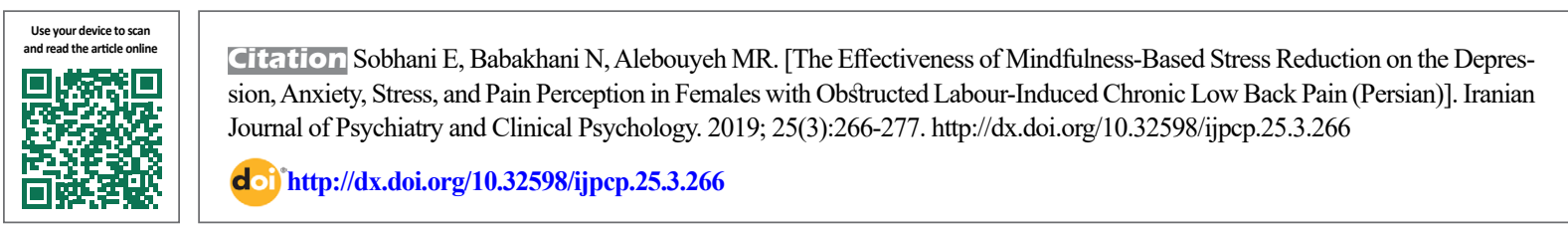

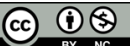

Received: 05 Sep 2018 Accepted: 07 May 2019 Available Online: 01 Oct 2019

Key words: Mindfulness-Based Stress Reduction (MBSR), Depression, Anxiety, Stress, Chronic low back pain

\begin{abstract}
A B S T R A C T
Objectives The present study aimed to investigate the effectiveness of Mindfulness-Based Stress Reduction (MBSR) on the severity of depression, anxiety, stress, and the perception of low back pain after childbirth.

Methods This was a quasi-experimental research with a pre-test, post-test and a control group and onemonth follow-up design. The statistical population included all females with chronic postpartum low back pain in Tehran City, Iran. Forty of them were selected by convenience sampling method and were assigned into the experimental (20) and control (20) groups. The study instruments included Depression Anxiety Stress Scale (DASS-21) and Ossouri's low back pain scale. The samples were followed-up one month after conducting the intervention. A mindfulness training program based on stress reduction was carried out in 8120 -minute sessions once a week for the experimental group. The collected data were analyzed using SPSS.

Results The study findings suggested a significant difference between the mean post-test scores of the experimental and control groups. Thus, mindfulness training on chronic low back pain perception had a significant effect at $P<0.01$. Additionally, mindfulness training had a significant effect on reducing depression, anxiety, and stress.

Conclusion Mindfulness exercises are associated with increasing the awareness of the thoughts, feelings, and senses of the patients and their admission. Moreover, these techniques reduced their level of depression, anxiety, stress, and lower chronic back pain perception. Therefore, they can help in designing better healthcare programs for therapists, counselors, and psychologists.
\end{abstract}

\section{Extended Abstract}

\section{Introduction}

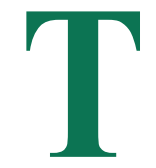

he high prevalence of low back pain during pregnancy and postpartum is among the most important issues worldwide. $\mathrm{Nu}$ merous women are extensively affected by chronic low back pain after pregnancy, and they fail to perform their daily and occupational activities. Thus, patients with chronic pain experience depression, stress, emotional disturbance, and fatigue. Psychological dimensions not only can shape emotional performance but also can affect nervous system activities through pain perception.

* Corresponding Author:

Narges Babakhani, PhD.

Address: Department of Psychology, Faculty of Psychology and Social Sciences, Roodehen Branch, Islamic Azad University, Roodehen, Iran.

Tel: +98 (21) 76505015

E-mail: babakhani@riau. ac.ir 
Thus, the maladaptive cognition of pain, like pain catastrophizing, may be accompanied by negative emotional and behavioral responses, such as stress, anxiety, and depression and impact the recovery of chronic pain. The mere use of medical methods, like pharmacotherapy, physiotherapy, etc. is not effective in the treatment of chronic lower back pain. Accordingly, new therapeutic pain-management approaches, like Mindfulness-Based Stress Reduction (MBSR) program are developed based on self-centered attention and self-focus; they can reduce pain by increasing the coping skills for chronic pain. In this method, the individual focuses on the process of focusing mind through teaching behavioral, cognitive, and metacognitive strategies. Thus, the present study aimed to investigate the effectiveness of MBSR on depression, anxiety, stress, and pain perception in women with delivery-induced chronic low back pain.

\section{Methods}

This was an experimental study with a pre-test, post-test design, control group, and one-month follow-up. The study population was all women with postpartum-induced chronic low back pain admitting to Saadat Abad Health Center in Tehran City, Iran, in 2017. Forty subjects were selected by random sampling method. They were randomly divided into the experimental (20 subjects) and control (20 people) groups. The study used Depression, Anxiety, and Stress Scale (DASS-21) by Lovibond \& Lovibond (1995) and Osteor in Patients with Inferiority Scale (ODI).

These questionnaires were used before and after performing the intervention and at one-month follow-up. The MBSR was derived from Kabat Zaynn's book (2013) and conducted in 8 sessions of 120 minutes for once a week on the experimental group. The session contents were as follows: first session: Setting up a general policy, relaxation training, discussion and appointment of weekly meetings, and distribution of educational pamphlets. Second session: Body-scan training.

Third session: Meditation training and review. Fourth session: Understanding mindful-breathing. Fifth session: Attention to body movements during breathing, focusing on body organs and their movements. Sixth session: Training paying attention to the mind, pleasant or unpleasant thoughts, allowing negative and positive thoughts to enter the mind and efficiently removing them from the mind. Seventh session: Seated meditating practice. Eighth session: Reviewing the past contents and summing up questions and answers. After the training completion, post-test was performed on both groups. Finally, the collected data were analyzed using SPSS.

\section{Results}

The mean scores of the dependent variables suggested significant differences between the experimental and control groups in the pre-test, post-test, and follow-up stages. This finding was in favor of the experimental group in all of the variables (understanding chronic back pain, depression, anxiety, and stress). The mixed variance analysis model was used to evaluate the effectiveness of mindfulnessbased stress reduction program. Accordingly, the necessary assumptions were examined to use the parametric test (mixed variance analysis). Considering the significance of Levene's test in the studied variables, the equality of variances was established.

The distribution of data was normal, the homogeneity of regression was slope, and the linearity of dependent and covariate regressions were observed. The mixed variance analysis results revealed a significant difference between the experimental and control groups. Given the obtained mean scores, this difference was in favor of the experimental group $(\mathrm{P}<0.05)$. Eta square obtained in the experimental group in all dependent variables was greater than 0.14 . Therefore, considering the significant interaction between the repetition of the test with the experimental variable and the magnitude of the effect obtained with a $99 \%$ confidence coefficient, MBSR could reduce chronic back pain perception, depression, anxiety, and stress in the experimental group, compared to the controls $(\mathrm{P}<0.05)$.

\section{Discussion}

The obtained results are consistent with those of Bakhshani et al. (2016), Dehestani (2015), Samadi et al. (2010), and Moroni et al. (2008). Mindfulness-training program focuses on in-person processes. Therefore, this technique can help individuals change their relationships with their inner states, thoughts, and emotions, and could reduce the internal anxiety symptoms. In MBSR, participants are encouraged to curiously observe their thoughts and feelings. They are also encouraged to consider a non-judgmental approach to their mental and emotional content, which reduces their depression.

Moreover, training MBSR techniques along with mental relaxation can lead to the normalization of respiration and the reduction of external symptoms of anxiety (palpitations, anxiety, pressure drop, sweating, and difficulty in breathing). Using relaxation techniques of MBSR returns the oxygen uptake of the body to a normal level, thereby increases the calmness and reduces the feeling of pain. As mindfulness exercises are associated with the increased awareness of patients, their thoughts, emotions, and body senses are 
associated with their acceptance, which reduces their level of depression, anxiety, stress, and perceived symptoms of chronic pain. The study signified the importance of mindfulness, the unpleasant consequences of stress, and chronic postpartum low back pain in women's lives. Thus, extensive activities and psychological interventions are essential and could be used to design better therapeutic programs by therapists, counselors, and psychologists.

\section{Ethical Considerations}

Compliance with ethical guidelines

All ethical principles were observed in this study and an ethical approval was obtained from the Research Ethics Committee of Islamic Azad University (Code: IR.IAU. TMU.REC.1396.217). Participants were free to leave the study at any time. They were aware of the study objective and method and were assured of the confidentiality of their information.

\section{Funding}

This study was extracted from a master thesis approved by Elahe Sobhani in the Faculty of Psychology and Social Sciences, at Islamic Azad University of Roodehen Branch.

Authors contributions

The authors had same contribution in preparing this paper.

\section{Conflicts of interest}

The authors declared no conflicts of interest. 


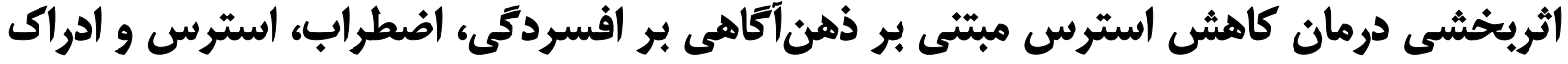

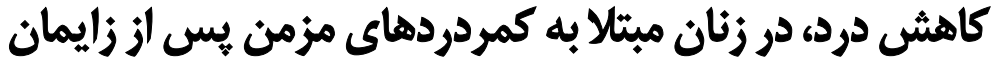

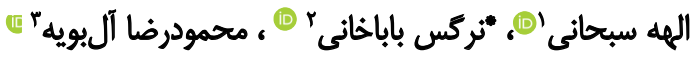 \\ ا - كارشناس ارشد روانشناسى، تروه روانشناسى، دانشكده روانشناسى و علوم اجتماعى، واحد رودهن، دانشعاه آزاد اسلامى، رودهن، ايران.

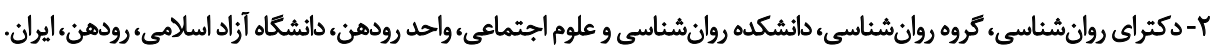

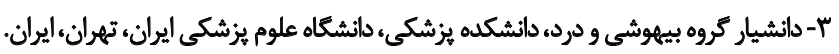

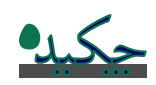

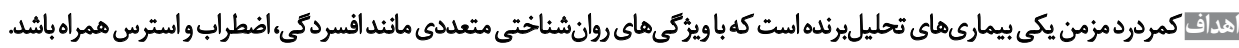

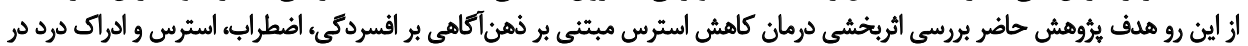

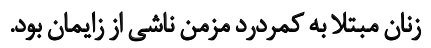

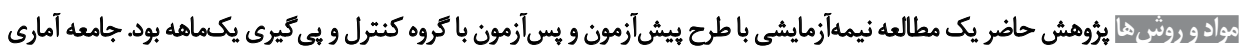

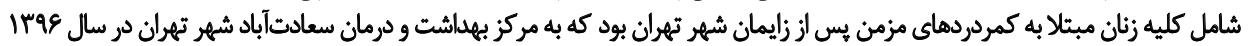

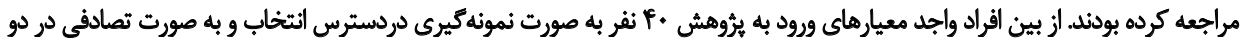

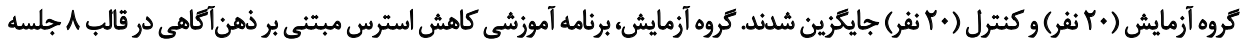

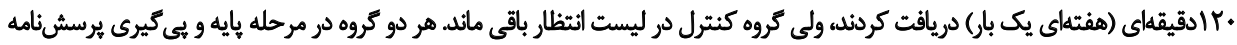

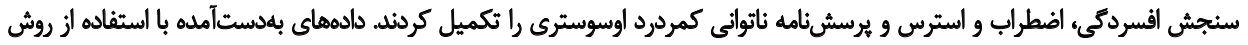

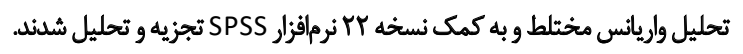

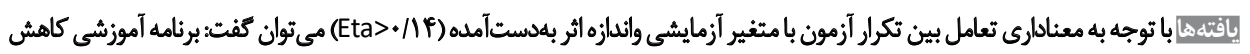

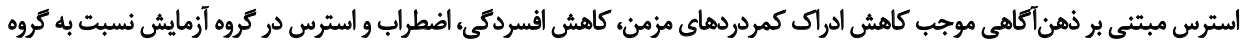

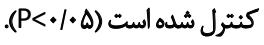

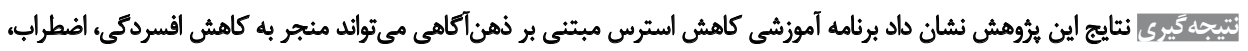

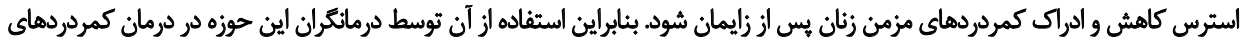
مزمن يس الز زايمان مؤثر است.
\end{abstract}

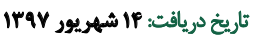

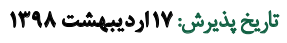

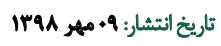

كليدوارةها:

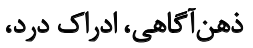

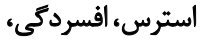

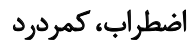

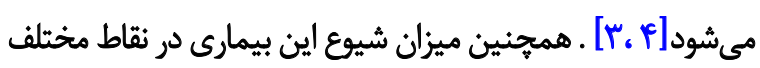

dale

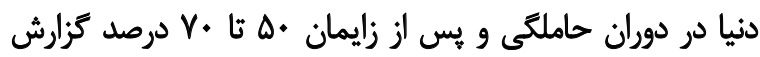

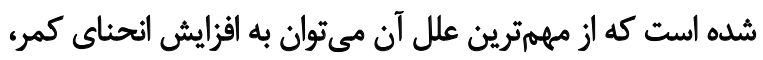

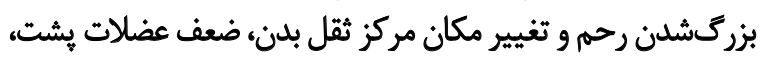

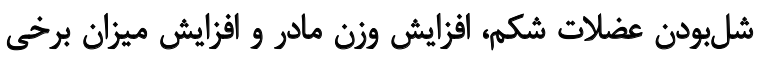

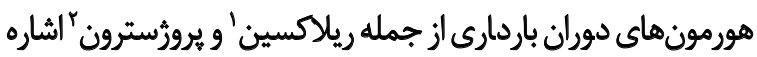

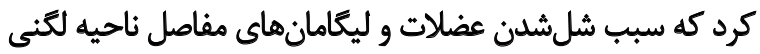

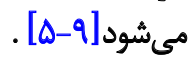

1. Relaxin

2. Progesterone

باردارى فرايند بيجيده فيزيولوزيكى است كه علاوه بر ايجاد

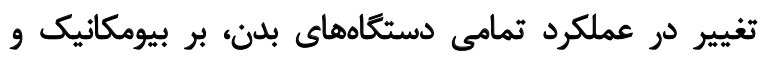

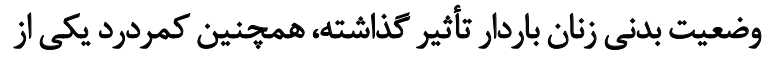

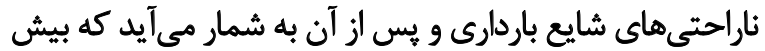

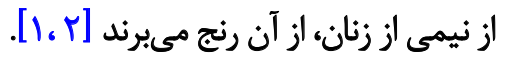

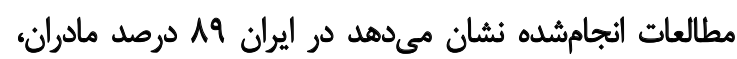

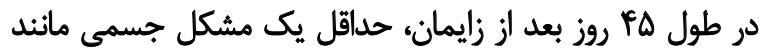

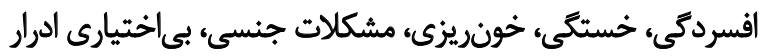

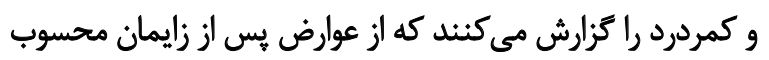

- 
كاهش استرس مبتنى بر ذهن آكاهي، در كاهش نشانهانهاي

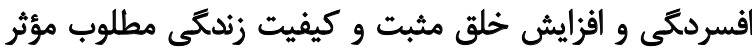

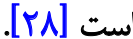

از طرف ديكر، ذهن آكاهي روى افراد با مشكلات مختلف

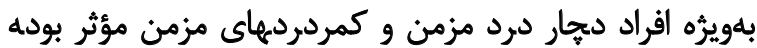

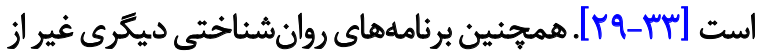

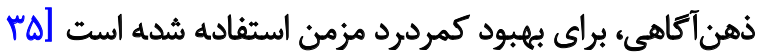

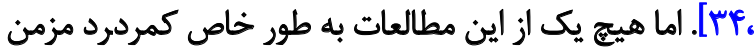

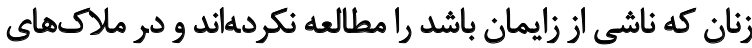

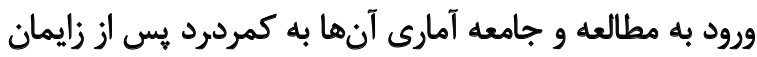

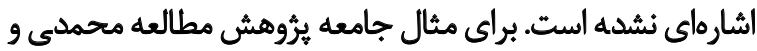

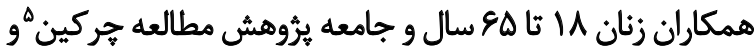

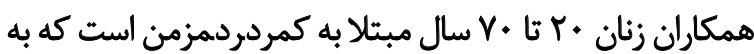

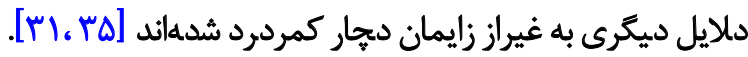

بنابراين با توجه به اثربخشى برنامه درمانى كاهش استرس

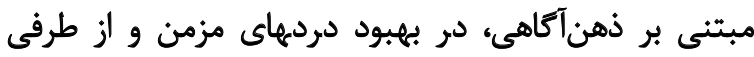

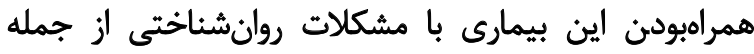

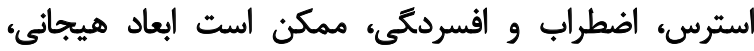

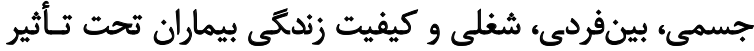

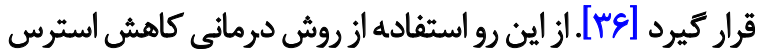

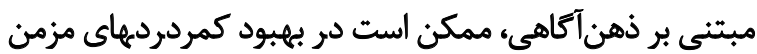

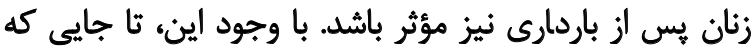

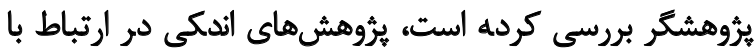

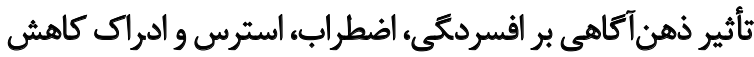

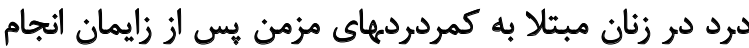

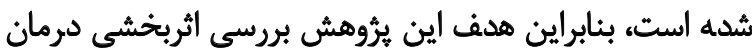

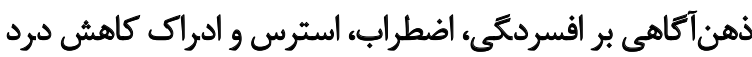

در زنان مبتلا به كمردرديهاى مزمن يس إز زايمان است إست

离

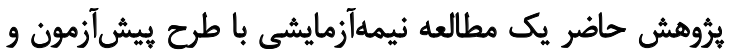

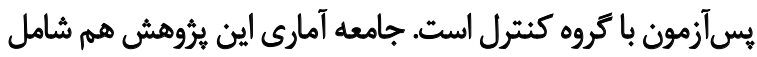

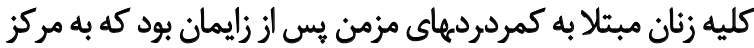

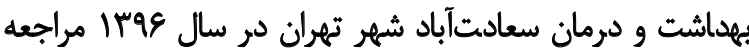

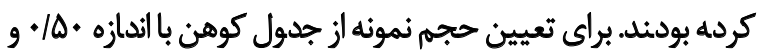

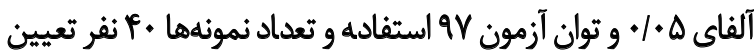

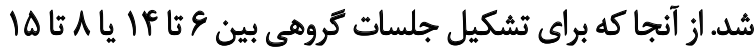

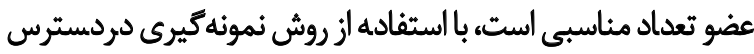

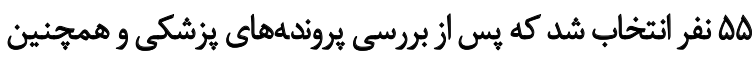

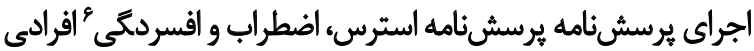

5. Cherkin

6. DASS-21
بروز و تداوم اين دردهاى مزمن بهويثه در طول دوران باردارى

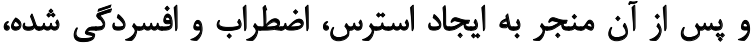

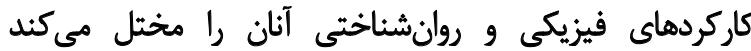

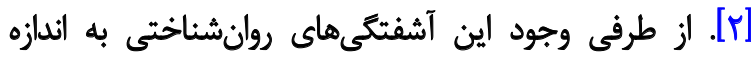

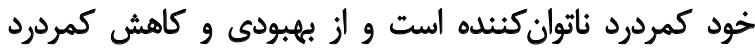

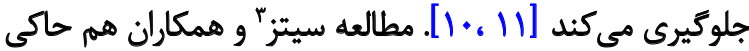

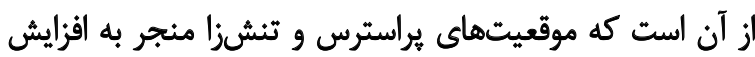

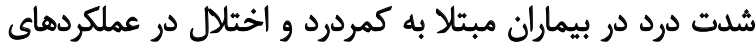

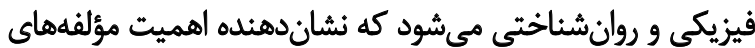

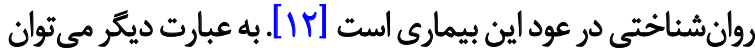

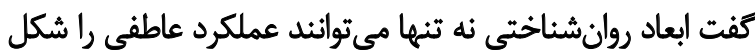

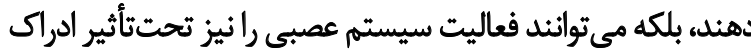

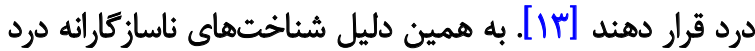

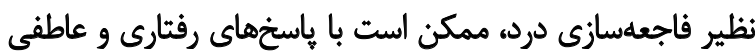

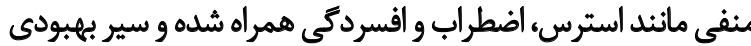

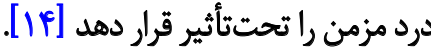

بررسى مطالعات صورتكرفته هم نشان مىدهد استفاده

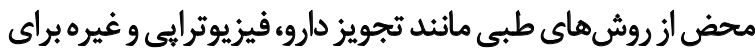

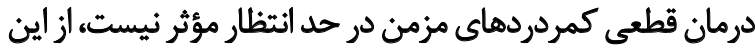

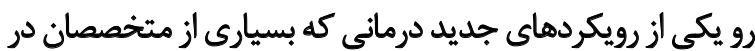

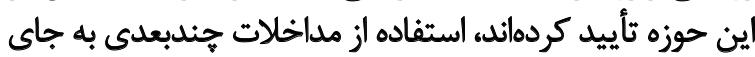

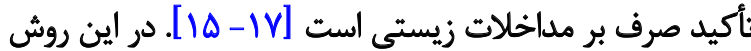

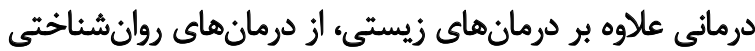

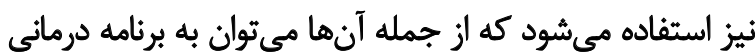

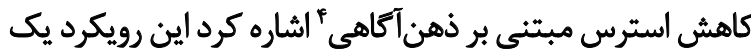

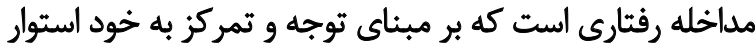

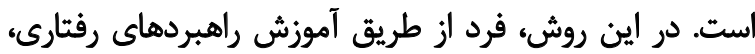

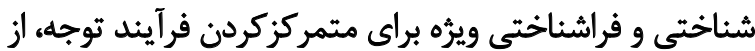

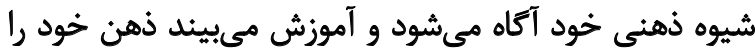

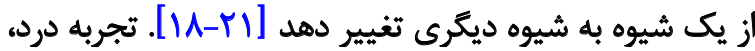

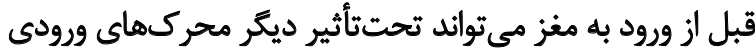

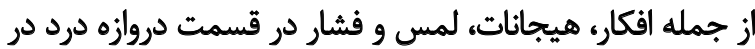

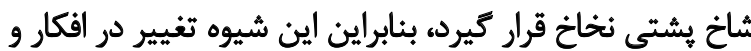

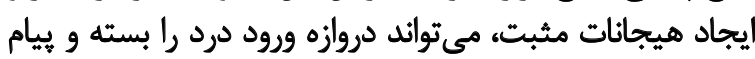

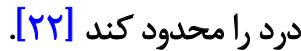

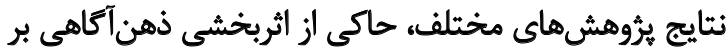

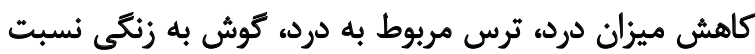

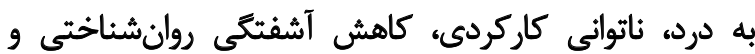

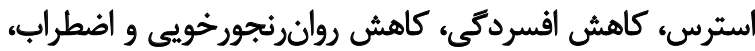

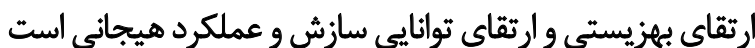

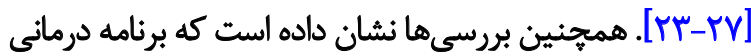

4. Mindfulness - Based Stress Reduction (MBSR) 


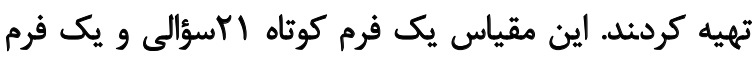

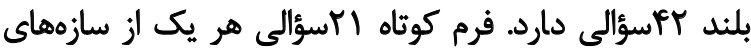

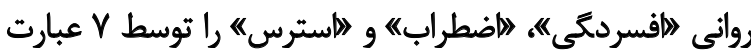

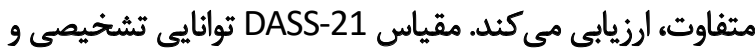

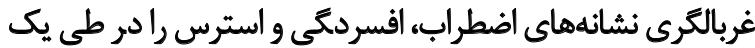

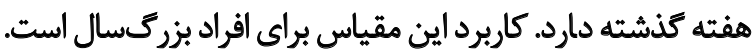

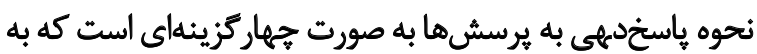

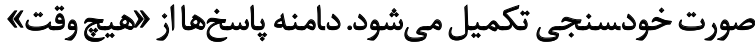

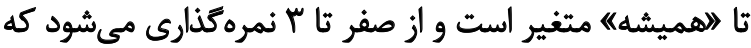

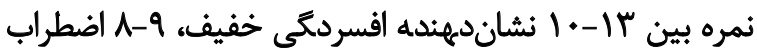

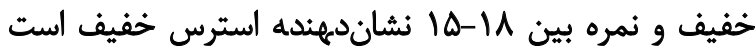

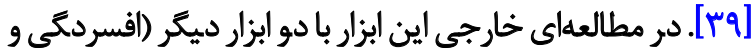

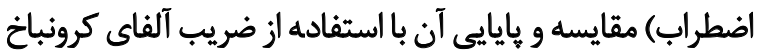

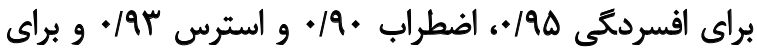

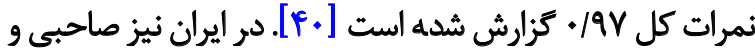

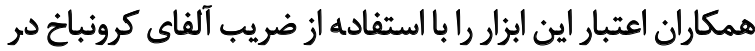

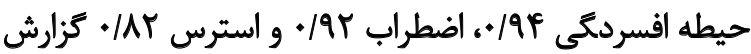

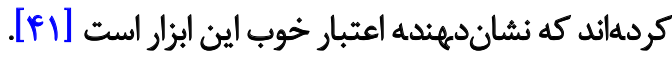
يرسش نامه ناتوانى اوسوسترى اين يرسشنامه شامل ده بخش

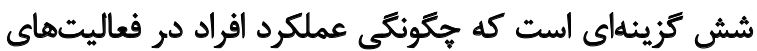

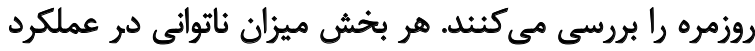

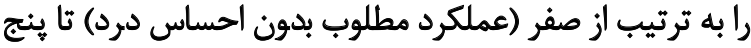

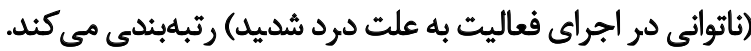

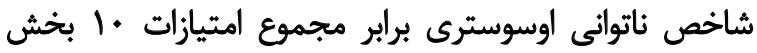

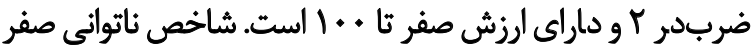

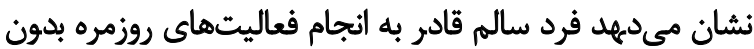

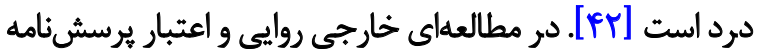

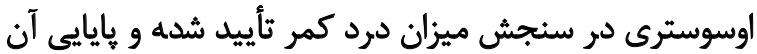

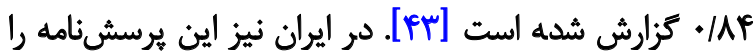

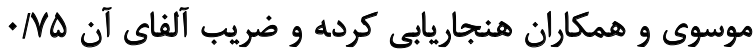

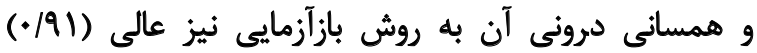

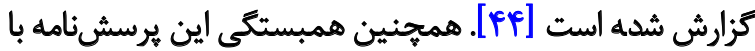

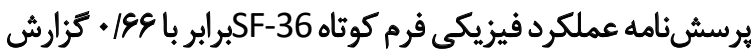
شده است.

\section{برنامه آموزشى مبتنى بر ذهن آكاهى}

در اين تحقيق از برنامه هشت جلسهاي ذهن آكاهي استفاده

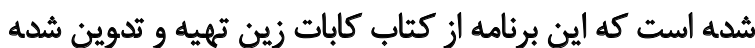

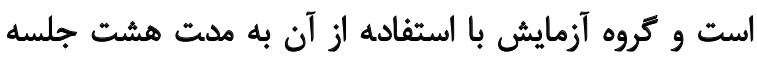

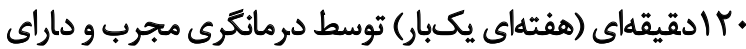

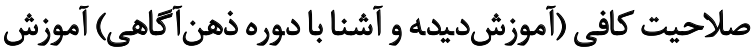

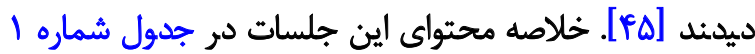
آورده شده است.

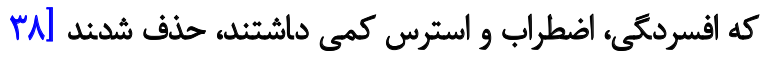

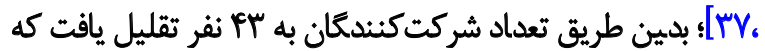

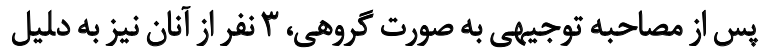

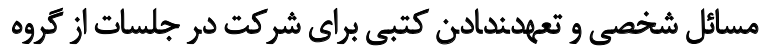

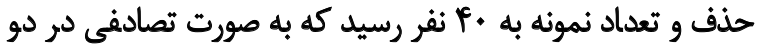

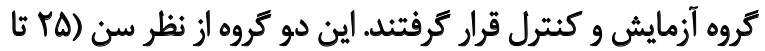

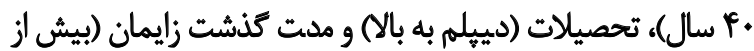

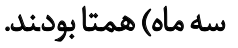

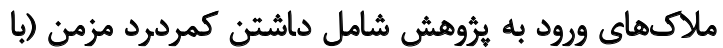

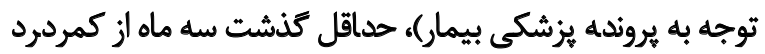

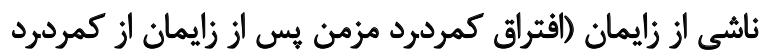

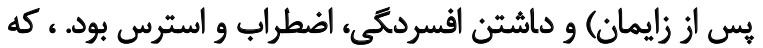

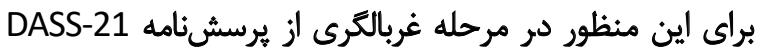

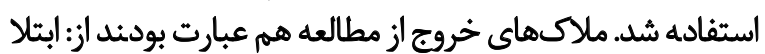

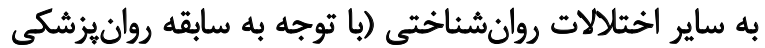

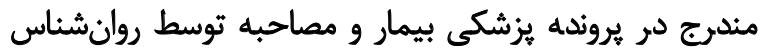

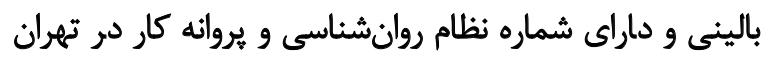

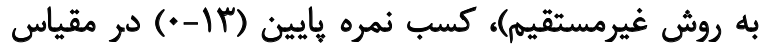

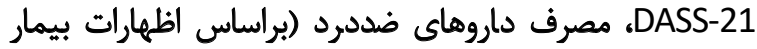

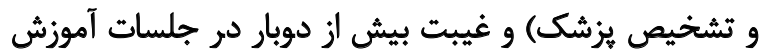

$$
\text { مديريت استرس مبتنى برذهن آكاهى بون ازيت دوبار }
$$

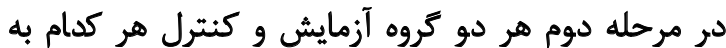

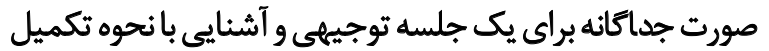

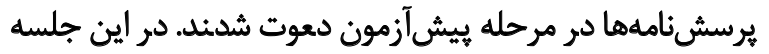

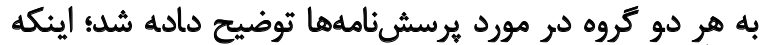

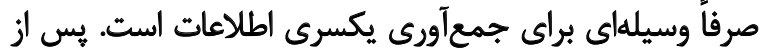

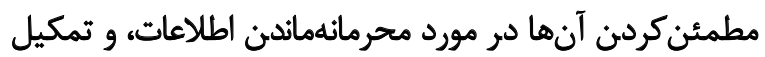

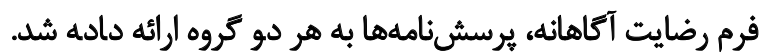

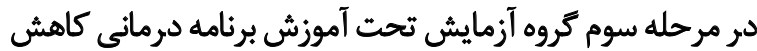

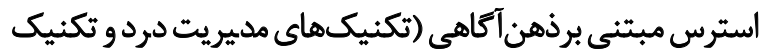

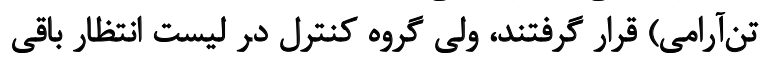

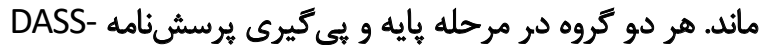

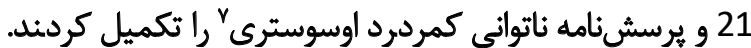

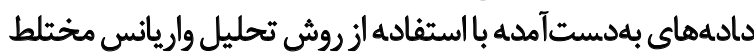

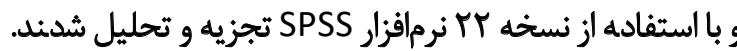

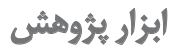

$$
\text { مقياس افسردَّى، اضطراب و استرس^ }
$$

اين يرسشنامه را در سال 1990 لاويبوند و لاويبوندج

7. Oswestry Disability Index (ODI)

8. Depression Anxiety and Stress Scale (DASS-21)

9. Lovibond \& Lovibond 
جدول ا. محتواى جلسات آموزشى با استفاده از برنامه كاهش استرس مبتنى بر ذهن آكاهيى

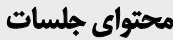
جلسه

تنظيم خط مشى كلى با درنظركرفتن جنبه محرمانهبودن و زندكى شخصى افراد، دعوت اقراد به معرفى خود با يكديكر، تمرين وارسى بلدنى، تكليف

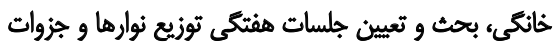

جلسه اول

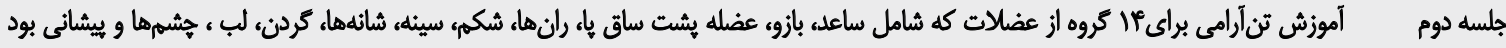
أموزش مراقبه و مرور جلسات كذشته

جلسه سوم

آشنايع با نحوه ذهن آكاهي تنفس، آموزش تكنيك دم و بازدم همراه آرامش و بلون تفكر در مورد جيز ديكر و آموزش تكنيك تماثاى تنفس و تكليف

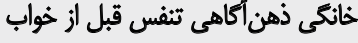

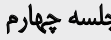

أهوزش تكنيك توجه به حركت بلن هنكام تنفس، تمركز بر اعضاى بلن و حركات أنها و جستوجوى حسهاى فيزيكى، تكليف خانكى ذهن آكاهى خون

جلسه هينجم

أموزش توجه به ذهن، افكار مثبت و منفى، خوشايند يا ناخوشايند بودن افكار، اجازددادن به ورود افكار منفى و مثبت به ذهن و بهآسانى خاجكردن آنها

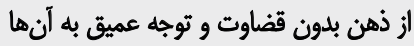

جلسه ثششم

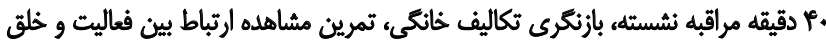

جلسه هفتم بازنكرى مطالب كذشته و جمعبندى و يرسش و ياسخ جلسيه هشتم

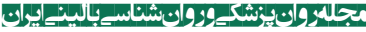

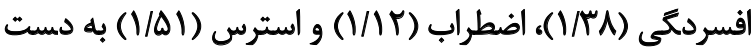

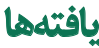

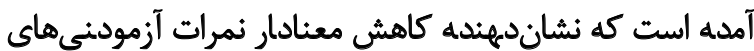

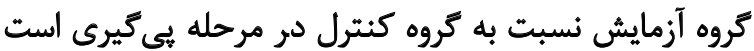

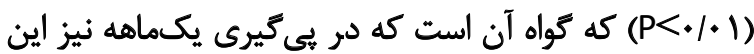
سير كاهش نمرات به طور معنى دارى حفظ شده است. براى بررسى ميزان اثربخشى برنامهاني درمانى كاهش استرس

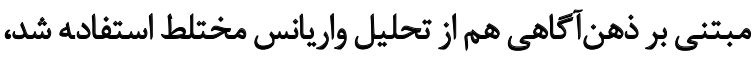

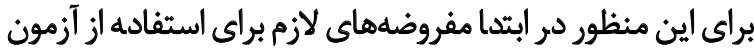

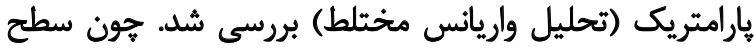

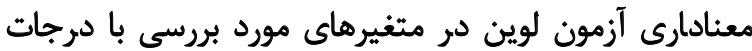

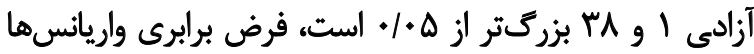

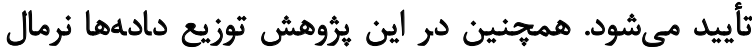

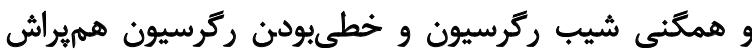

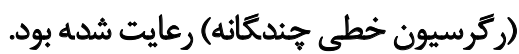

نتايج حاصل از تحليل واريانس مختلط نشان داد بين گروره

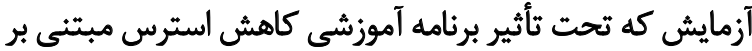

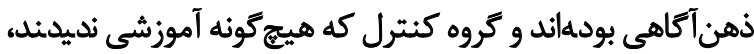

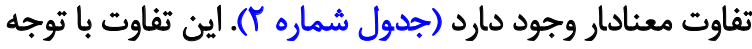

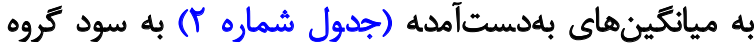

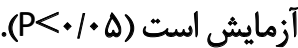

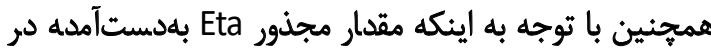

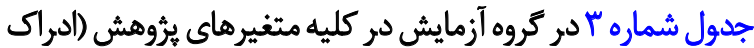

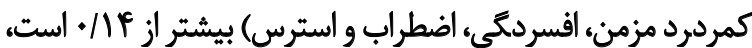

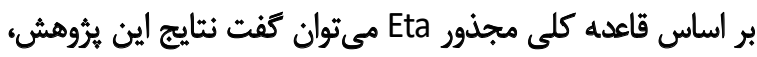

نتايج بلهستآمده از اطلاعات جمعيتشناختى زنان دان داروائ

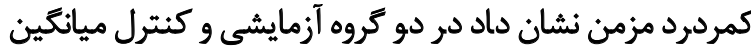

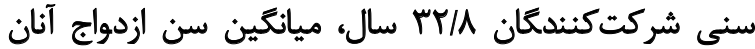
Y9/99

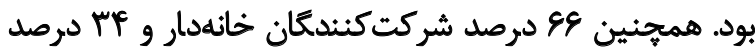

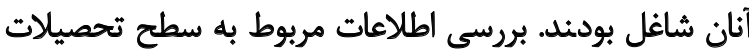

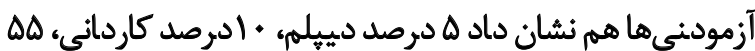

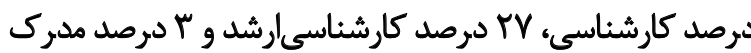
تحصيلى دكترى داشتئد.

همجنين درجدول شماره Y ميانگين و انحراف استاندارد

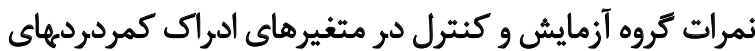

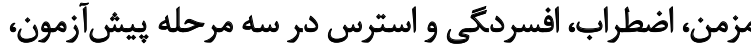

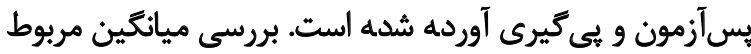

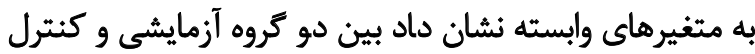

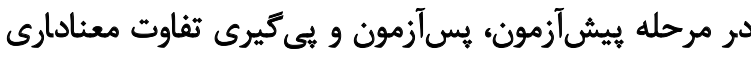

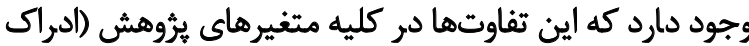

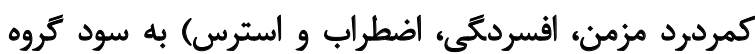

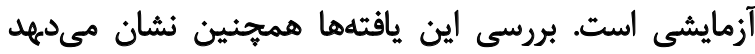

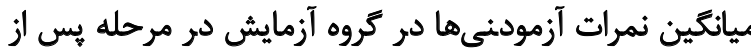

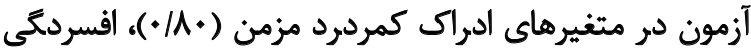

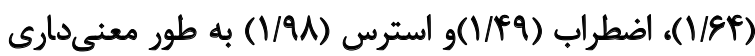

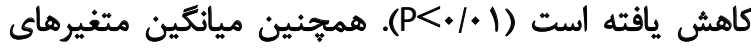

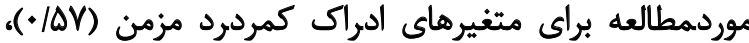


جدول r. شاخصهاى توصيفى نمرات ادراك كمردردهاى مزمن، استرس،اضطراب و افسرديى در سه مرحله اندازمكيرى براى كروه آزمايش و كتترل.

\begin{tabular}{|c|c|c|c|}
\hline \multicolumn{2}{|c|}{ ميانكيند|نحراف معيار } & \multirow{2}{*}{\multicolumn{2}{|c|}{ متغيرها }} \\
\hline كروه كتترل & كروه أزمايش & & \\
\hline V/gYt+/gV & $V / \& \Delta \pm+/ F F$ & ييشأزمون & \\
\hline$V / A r \pm+/ 9 V$ & $\cdot / \Lambda \cdot \pm+/ \pi T$ & يس آزمون & ادراك كمردردهاى مزمن \\
\hline$r / .9 \pm+/ 9 q$ & $. / \Delta Y \pm+/ \Delta$. & ييكيرى & \\
\hline$T / I F \pm . / M$ & $r I \pm . r \cdot / \Lambda \Delta$ & ييش آزمون & \\
\hline$r / 8 \cdot \pm \cdot / \Lambda$. & V/gF士./9V & قِيسأزمون & افسردمى \\
\hline$r / q) \pm \cdot / \% \Delta$ & $1 / \% A \pm \cdot / \% 8$ & ييكيرى & \\
\hline$V / A E \pm=/ A V$ & $1 / W \pm \cdot / A r$ & يشئزمون & \\
\hline$r / M \pm \cdot / \pi T$ & 1/eq土./9\% & يس آزمون & اضطراب \\
\hline$r / .9 \pm . / 99$ & I/TYE./Ar & ييكيرى & \\
\hline$r / \Delta P \pm=/ S A$ & $r / \Delta f \pm \cdot N E$ & ييشآزمون & \\
\hline$r / M \Psi \pm / \Delta V$ & $1 / 4 \pm . / 91$ & ي يسآزمون & اسثرس \\
\hline$r / r \Psi \pm=/ p q$ & $V / \Delta I \pm \cdot / P A$ & ييكيرى & \\
\hline
\end{tabular}

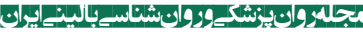

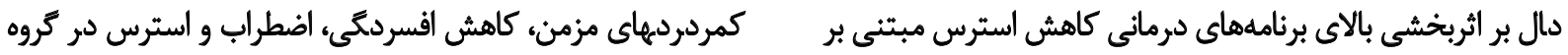

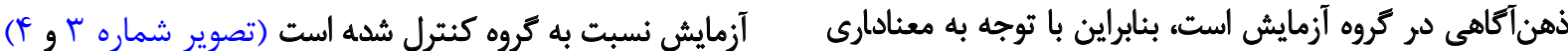

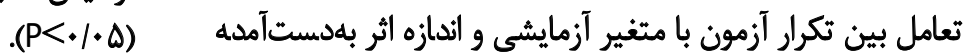

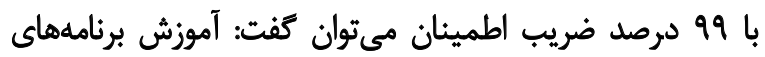

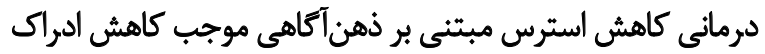

جدول r. آزمون تجزيه و تحليل واريانس مختلط ادراك كمردرد مزمن، افسرديى، اضطراب و استرس

\begin{tabular}{|c|c|c|c|c|c|c|}
\hline الندازه اثر & معنى دطحى & $\mathrm{F}$ & MS & منابع تغيير & & مثغير \\
\hline.$/ r V$ & $.1 \cdot 1$ & $r / \Delta)$ & V/AY & آزمون (تكرار اثندازةكيرى) & \multirow{2}{*}{ درون كروهى } & \multirow{3}{*}{ ادراك كمردرد مزمن } \\
\hline$\cdot / \pi$ & .1 .1 & $r Y / Q)$ & V/ar & تعامل آزمون با متغير آزمايشى & & \\
\hline.$/ 49$ & $+1+1$ & $r F / M^{e}$ & $r / / Y r$ & متغير آزمايش & يينكروهى & \\
\hline .1 .1 &.$/ 199$ &.$/ 19$ & .1 .4 & آزمون (تكرار اندازهكيرى) & \multirow{2}{*}{ درون كروهى } & \multirow{3}{*}{ افسردكى } \\
\hline.$/ A T$ & .1 .1 & $P 1 / T A$ & V/AT & تعامل آزمون با متغير آزمايشى & & \\
\hline$\cdot|r|$ & .1 .1 & IV/TA & $19 / 4$ & متغير آزمايش & بين كروهى & \\
\hline.$M$ & $.1 . Y$ & P/AS &.$/ 09$ & آزمون (تكرار اثلدازهيرى) & \multirow{2}{*}{ درون كروهى } & \multirow{3}{*}{ اضطراب } \\
\hline.$/ \% \Delta$ & .1 .1 & $r \cdot / M r$ & $r / P Q$ & تعامل آزمون با متغير آزمايشى & & \\
\hline 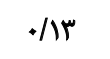 & $\%$ & $\Delta / A r$ & NI9 & متفير آلزمايش & يبن كروهى & \\
\hline.$/ 10$ & .1 .1 & $8 / p q$ & .108 & آزمون (تكرار اندازةكيرى) & \multirow{2}{*}{ درون كروهى } & \multirow{3}{*}{ استرس } \\
\hline . Na & .1 .1 & $11 T / r$ & Q/बV & تعامل آزمون با متغير آزمايشى & & \\
\hline (צוא/. & $.1+1$ & $r / M$ & $r \cdot / \& A$ & متغير آزمايش & يين كروهى & \\
\hline
\end{tabular}

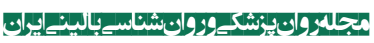




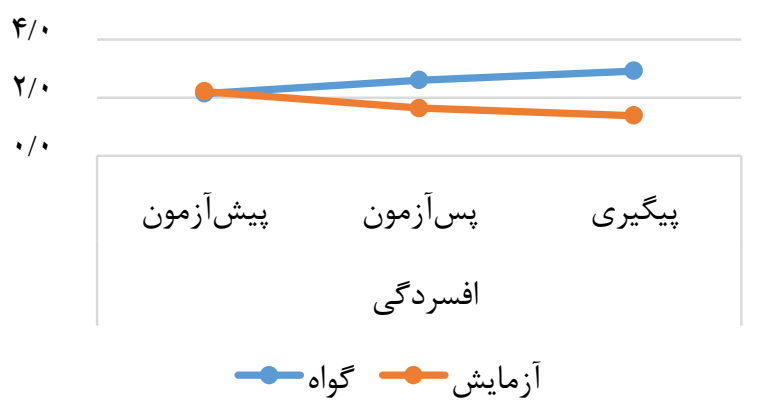

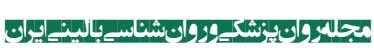

تصوير r. مقايسه ميائكين افسردكى در سه مرحله اندازهيرى براي كروههاى موردمطالعه

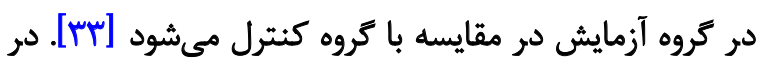

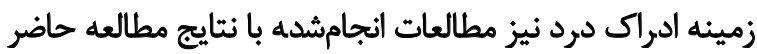

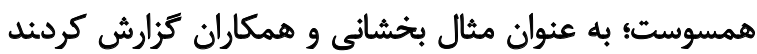

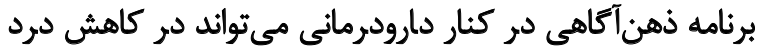

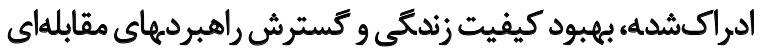

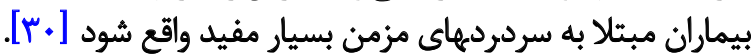

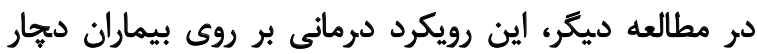

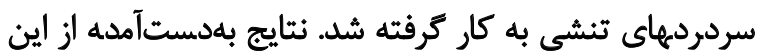

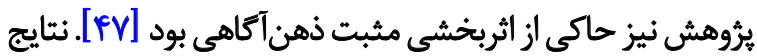

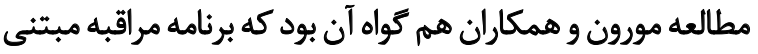

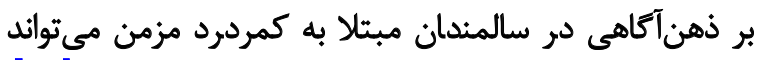

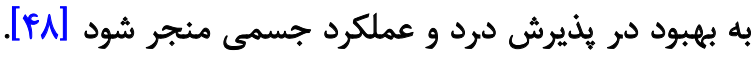

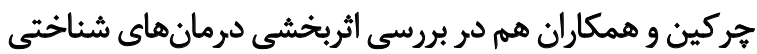

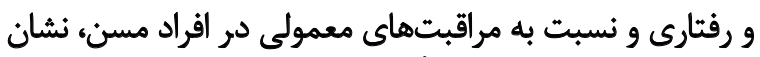

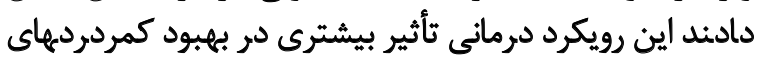

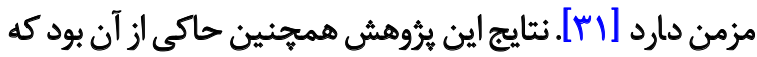

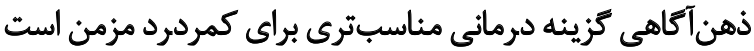

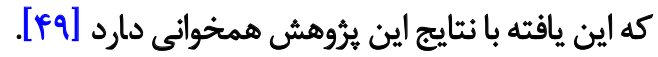

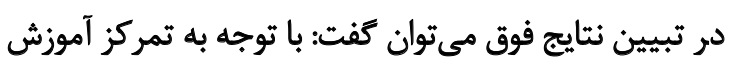

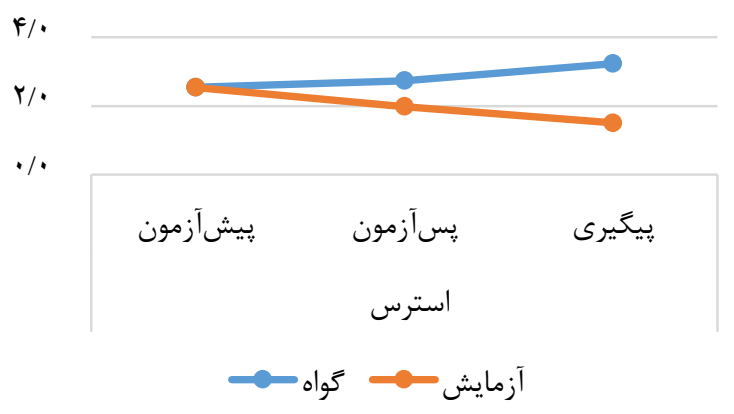

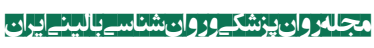

تصوير F. مقايسه ميانكين استرس در سه مرحله اندازهكيرى براى كروههاي

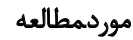

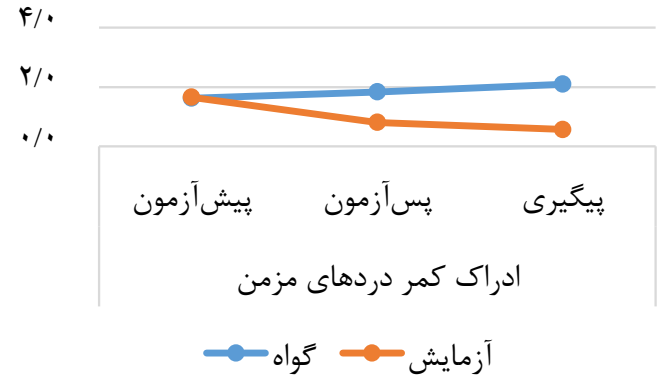

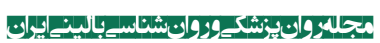

تصوير ا. مقايسه ميانكين ادراك كمردرديهاى مزمن در سه مرحله اندازهكيرى براى كروههاي موردمطالعه

\section{ب}

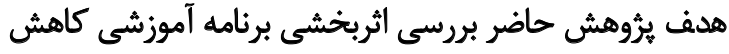

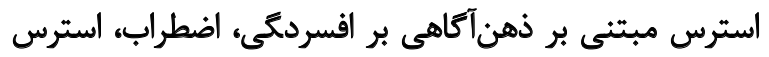

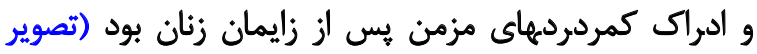

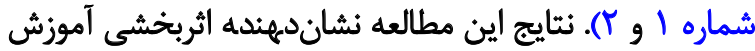

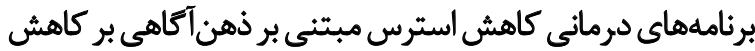

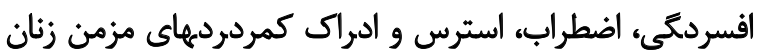
مبتلا به كمردردهاي مزمن يس از زايمان بود (تصوير شماره جائ).

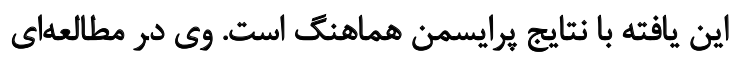

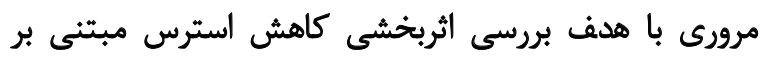

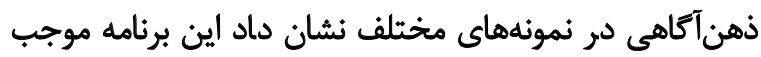

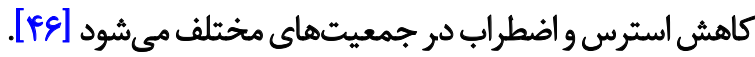

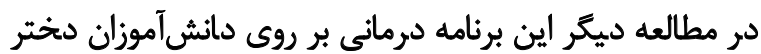

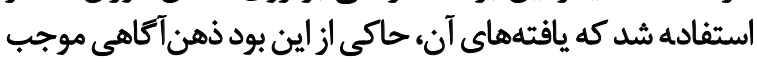

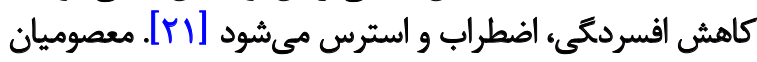

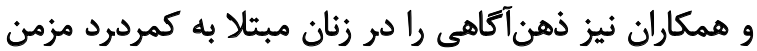

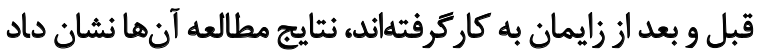

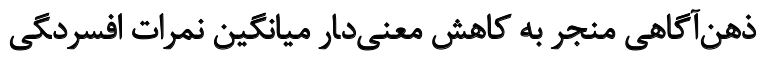

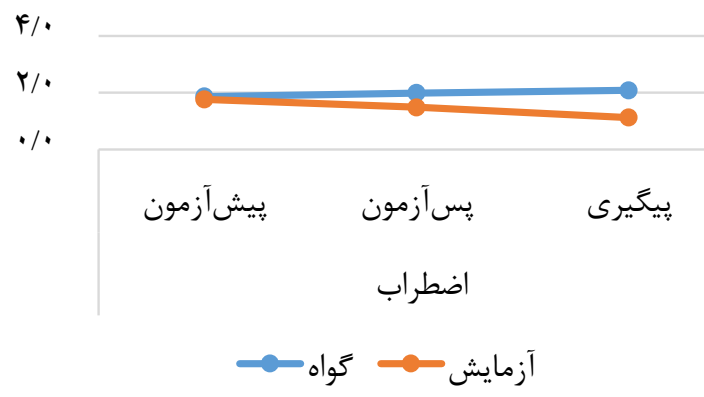

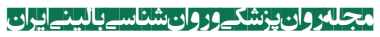

تصوير ז. مقايسه ميائكين اضطراب در سه مرحله اندازمكيرى براي كروههاى

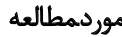


متفاوت مكانيسمهاي رواني مقابله با درد نسبت به زنان هستئد)

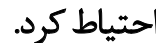

\section{مالاحظات اخلاقى}

\section{ييروى از الصول الخاق يُوهش}

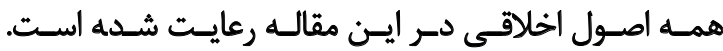

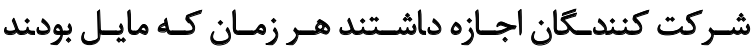

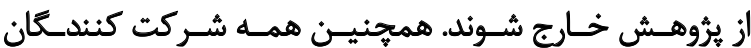

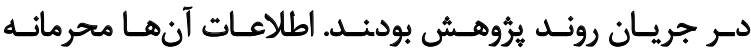

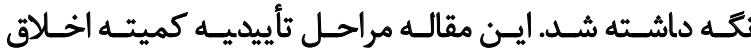

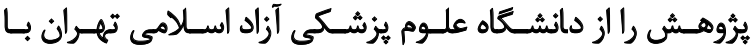

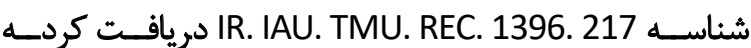
استـت. است.

$$
\text { مامي مالب }
$$

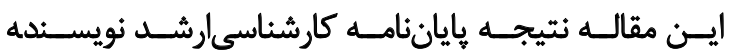

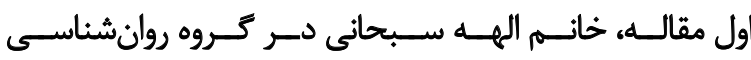

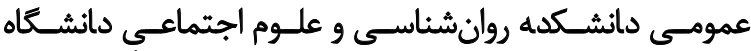

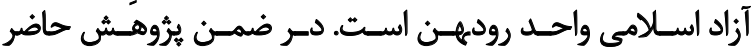

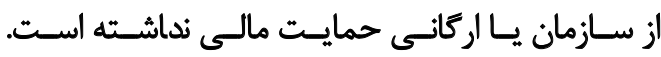

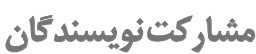

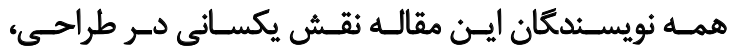

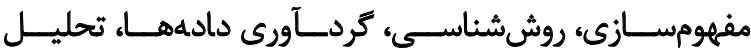

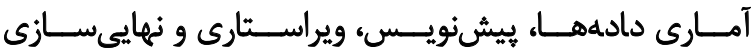
داشـــــاند.

تعارض منافُع

طبق نظر نويسندكان اين مطالعه هيجگونه تضاد منافعى

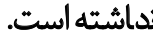

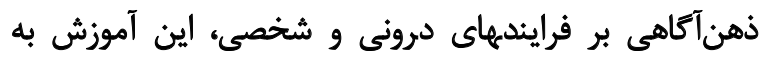

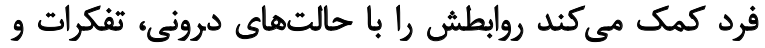

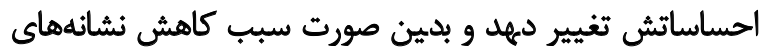

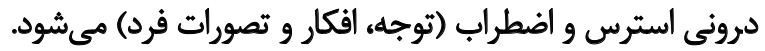

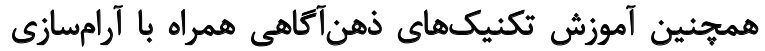

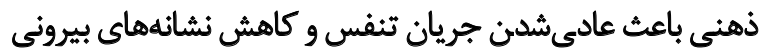

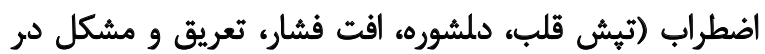

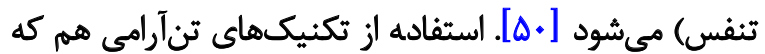

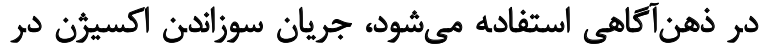

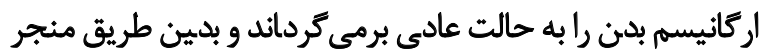

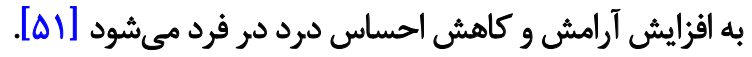
از طرفى در برنامهاى آموزشى مبتنى بر ذهنآتاهيى"،

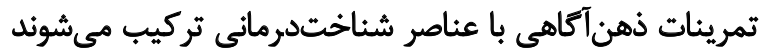

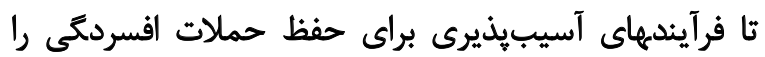

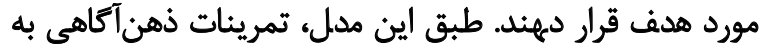

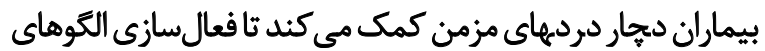

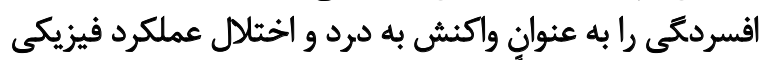

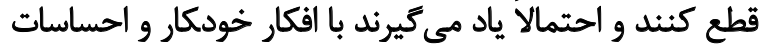

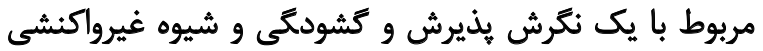

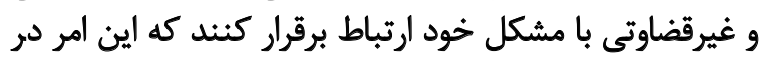

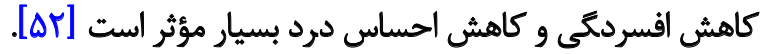

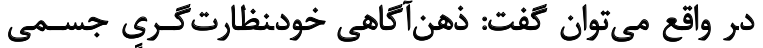

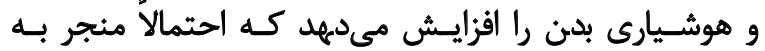

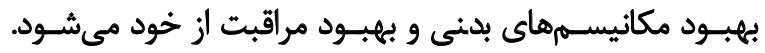

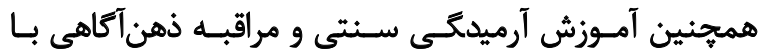

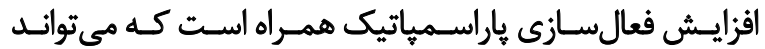

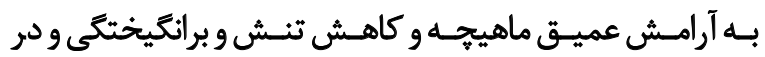

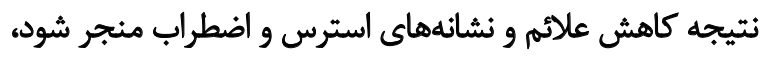

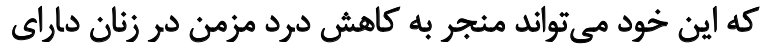

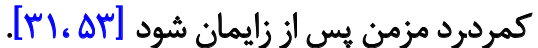

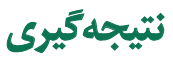

نتايج اين يُروهش نشان داد برنامه آموزشى كاهش استرس

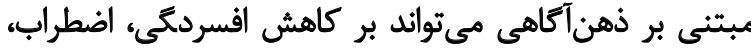

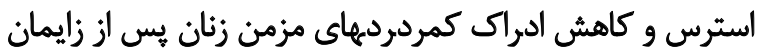

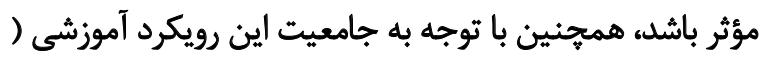

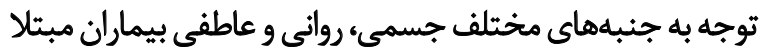

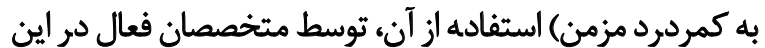

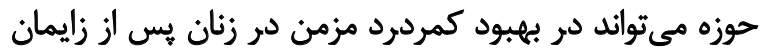

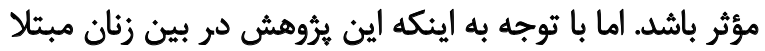

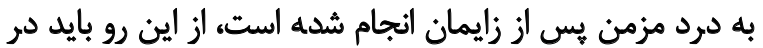

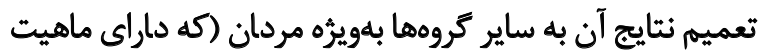

10. Mindfulness- Based Cognitive Therapy (MBCT) 


\section{References}

[1] Close C, Sinclair M, Liddle SD, Madden E, McCullough JE Hughes C. A systematic review investigating the (LBPP) in pregnancy. Journal of Advanced Nursing. 2014; 70(8):1702-16. [DOI:10.1111/jan. 12360] [PMID]

[2] Zahedpour F, Mohammadi M, Damavandi M, Agah J. [The effect of core stability training on postpartum lumbar lordosis and low back pain in nulliparous women (Persian)]. The Iranian Journal of Obstetrics, Gynecology and Infertility. 2017; 20(3):89-97. [DOI:10. 22038/IJOGI. 2017. 8876]

[3] Roohi M. [Maternal morbidity within 45 days after delivery (Persian)]. Iran Journal of Nursing. 2005; 18(41):145-52.

[4] Abedini Z, Nikpour M, Mokhber NA, Ebrahim SA, Khani SO. [Evaluation of relationship between delivery mode and postpartum quality of life (Persian)]. The Iranian Journal of Obstetrics, Gynecology and Infertility. 2010; 3(13):47-53.

[5] Mohseni Bandpei MA, Fakhri M, Âhmad Shirvani M, Bagheri Nesami M. [Low back pain in pregnancy: A systematic review of the literature (Persian)]. Journal of Mazandaran University of Medical Sciences. 2004; 14(44):94-107.

[6] Tsang A, Von Korff M, Lee S, Alonso J, Karam E, Angermeyer $\mathrm{MC}$, et al. Common chronic pain conditions in developed and developing countries: Gender and age differences and comorbidity with depression-anxiety disorders. The Journal of Pain. 2008; 9(10):883-91. [DOI:10. 1016/j.jpain. 2008. 05. 005] [PMID]

[7] Sedaghati P, Daneshmandi H, Saremi A. T, Ashtari M. [Effect of eight weeks aquatic gymnastics training on the intensity of low back pain in primiparous women (Persian)]. Journal of Sabzevar University of Medical Sciences. 2018; 25(3):279-86.

[8] Sabino J, Grauer JN. Pregnancy and low back pain. Current reviews in musculoskeletal -medicine. 2008; 1(2):137-41 [DOI:10. 1007/s12178-008-9021-8] [PMID] [PMCID]

[9] To W, Wong M. Factors associated with back pain symptoms in pregnancy and the persistence of pain 2 years after pregnancy. Acta Obstetricia et Gynecologica Scandinavica. 2003; 82(12):1086-91. [DOI:10. 1046/j. 1600-0412. 2003. 00235. x] [PMID]

[10] Jonbozorgi M, Golchin N, Alipour A, Agah Heris M. [The effectiveness of group cognitive-behavior therapy on decreasing severity of pain and psychological distressamong women with chronic back pain (Persian)]. Iranian Journal of Psychiatry and Clinical Psychology. 2013; 19(2):102-8.

[11] Cunningham G, leveno J, Bloom S, Hauth J, Spong C. Williams obstetrics. New York: Access Medicine; 2010.

[12] Seitz DC, Besier T, Debatin KM, Grabow D, Dieluweit U, Hinz A, et al. Posttraumatic stress, depression and anxiety among adult long-term survivors of cancer in adolescence. European Journal of Cancer. 2010; 46(9):1596-606. [DOI:10. 1016/j. ejca. 2010. 03. 001] [PMID]

[13] Seminowicz DA, Davis KD. A re-examination of pain-cognition interactions: Implications for neuroimaging. Pain. 2007; 130(1):813. [DOI:10.1016/j. pain. 2007. 03. 036] [PMID]

[14] Soleimani Sefat E, Sa'adati H, Azimian S, Amani S, Leshni L. Psychometric properties of Persian Version of Young-Rygh Avoidance Inventory. Iranian Journal of Psychiatry and Clinical Psychology. 2017; 23(2):232-43. [DOI:10. 29252/nirp. ijpcp. 23. 2. 232]
[15] Nicholas M, Molloy A, Beeston L, Tonkin L. Manage your pain Practical and positive ways of adapting to chronic pain. London: Souvenir Press; 2012.

[16] Nilsson-Wikmar L, Holm K, Öijerstedt R, Harms-Ringdahl K. Effect of three different physical therapy treatments on pain and activity in pregnant women with pelvic girdle pain: A randomized clinical trial with 3, 6, and 12 months follow-up postpartum. Spine. 2005; 30(8):850-6. [DOI:10. 1097/01. brs. 0000158870 68159. d9] [PMID]

[17] Stuge B, Hilde G, Vøllestad N. Physical therapy for pregnancy-related low back and pelvic pain: A systematic review. Acta Obstetricia et Gynecologica Scandinavica. 2003; 82(11):983-90. [DOI:10. 1034/j. 1600-0412. 2003. 00125. x] [PMID]

[18] Shapero BG, Greenberg J, Pedrelli P, Desbordes G, Lazar SW. Mindfulness-based cognitive therapy. In: Shapero B, Mischoulon D, Cusin C, editors. Massachusetts General Hospital Guide to Depression. Berlin: Springer; 2019. [DOI:10. 1007/978-3-31997241-1_13]

[19] Crane R. Mindfulness based cognitive therapy: The CBT distinctive features series. Abingdon: Routledge; 2009. [DOI:10. 4324/9780203882344]

[20] Michalak J, Burg J, Heidenreich T. Don't forget your body: Mindfulness, embodiment, and the treatment of depression Mindfulness. 2012; 3(3):190-9. [DOI:10. 1007/s12671-012-0107-4]

[21] Dehestani M. [The effectiveness of mindfulness-based stress reduction program on depression, anxiety and depression of female students (Persian). Journal of Thought \& Behavior in Clinical Psychology. 2015; 10(37):47-56.

[22] Zareh H, Mohammadi N, Moghighi P, Afshar H, Pourkazem L. [The effect of adjusted cognitive therapy based on mindfulness on disaster recognition, acceptance and pain severity in patients with fibromyalgia (Persian)]. Iranian Journal of Research in Psychology. 2015; 3(4):97-115.

[23] Schütze R, Rees C, Preece M, Schütze M. Low mindfulness predicts pain catastrophizing in a fear-avoidance model of chronic pain. Pain. 2010; 148(1):120-7. [DOI:10. 1016/j. pain. 2009. 10. 030] [PMID]

[24] Carmody J, Baer RA. Relationships between mindfulness practice and levels of mindfulness, medical and psychological symptoms and well-being in a mindfulness-based stress reduction program. Journal of Behavioral Medicine. 2008; 31(1):23-33. [DOI:10 1007/s10865-007-9130-7] [PMID]

[25] Sanders WA, Lam DH. Ruminative and mindful self-focused processing modes and their impact on problem solving in dysphoric individuals. Behaviour Research and Therapy. 2010; 48(8):747-53. [DOI:10. 1016/j. brat. 2010. 04. 007] [PMID]

[26] Brown KW, Ryan RM. The benefits of being present: Mindfulness and its role in psychological well-being. Journal of Personality and Social Psychology. 2003; 84(4):822. [DOI:10. 1037/0022 3514. 84. 4. 822]

[27] Gardner-Nix J. Mindfulness-based stress reduction for chronic pain management. In Didonna F, editor. Clinical handbook of mindfulness. New York: Springer; 2009. [DOI:10. 1007/978-0-38709593-6_20]

[28] Nyklíček I, Vingerhoets AD, Zeelenberg M. Mindfulness, emotion regulation, and well-being. Berlin:Springer; 2011. [DOI:10 1007/978-1-4419-6953-8_7] 
[29] Kabat-Zinn J, Lipworth L, Burney R. The clinical use of mindfulness meditation for the self-regulation of chronic pain. Journal of Behavioral Medicine. 1985; 8(2):163-90. [DOI:10. 1007/ BF00845519] [PMID]

[30] Bakhshani NM, Amirani A, Amirifard H, Shahrakipoor M. The effectiveness of mindfulness-based stress reduction on perceived pain intensity and quality of life in patients with chronic headache. Global Journal of Health Science. 2016; 8(4):142-51. [DOI:10. 5539/gihs. v8n4p142] [PMID] [PMCID]

[31] Cherkin DC, Sherman KJ, Balderson BH, Cook AJ, Anderson ML, Hawkes RJ, et al. Effect of mindfulness-based stress reduction vs cognitive behavioral therapy or usual care on back pain and functional limitations in adults with chronic low back pain: A randomized clinical trial. JAMA. 2016; 315(12):1240-9. [DOI:10. 1001/jama. 2016. 2323] [PMID] [PMCID]

[32] Morone NE, Greco CM, Weiner DK. Mindfulness meditation for the treatment of chronic low back pain in older adults: A randomized controlled pilot study. Pain. 2008; 134(3):310-9. [DOI:10. 1016/j. pain. 2007. 04. 038] [PMID] [PMCID]

[33] Samadi H, Rajabi R, Mlinoonejad h, Shahi Y, Samadi F. [Comparison the rate of pain, disability and psychological symptoms in female patients with chronic low back pain pre and post stabilization training (Persian)]. Olympic Journal. 2010; 18(2):25-34.

[34] Vakili N, MA ND. [The effect of cognitive-behavioral group pain management therapy on depression of the female with chronic low back pain (Persian)]. Journal of Clinical Psychology. 2009; 1(4):11-9.

[35] Mohammadi F, Imanzad M, Mohammadkhani P, Dolatshahi B, ali Asghari M, Tavassoli E. The effectiveness of "mindfulness based cognitive therapy" on psycho-social performance of Chronic Low Back Pain patients (CLBP). Advances in Nursing \& Midwifery. 2014; 23(82):46-54.

[36] Simpson AK, Cholewicki J, Grauer J. Chronic low back pain. Current Pain and Headache Reports. 2006; 10(6):431-6. [DOI:10. 1007/s11916-006-0074-3] [PMID]

[37] Corey MS, Corey G, Corey C. Groups: Process and practice. Boston: Cengage Learning; 2013.

[38] Soleimani Sefat E, Younesi SJ, Dadkhah A, Rostami M. Effectiveness of cognitive behavioral therapy training in reducing depression in visually impaired male students. Iranian Rehabilitation Journal. 2017; 15(2):165-72. [DOI:10. 18869/nrip. irj. 15. 2. 165]

[39] Lovibond S, Lovibond P. Manual for the depression anxiety stress scales. Sydney: Psychology Foundation of Australia; 1995. [DOI:10. 1037/t01004-000]

[40] Crawford JR, Henry JD. The Depression Anxiety Stress Scales (DASS): Normative data and latent structure in a large non-clinical sample. British Journal of Clinical Psychology. 2003; 42(2):11131. [DOI:10. 1348/014466503321903544] [PMID]

[41] Sahebi A, Asghari MJ, Salari RS. [Validation of Depression Anxiety and Stress Scale (DASS-21) for an Iranian population (Persian)]. Iranian Psychologists. 2005; 4(1):299-313.

[42] Fairbank JC, Pynsent PB. The Oswestry disability index. Spine. 2000; 25(22):2940-53. [DOI:10. 1097/00007632-200011150-00017] [PMID]

[43] Davidson M, Keating JL. A comparison of five low back disability questionnaires: Reliability and responsiveness. Physical Therapy. 2002; 82(1):8-24. [DOI:10. 1093/ptj/82. 1. 8] [PMID]
[44] Mousavi SJ, Parnianpour M, Mehdian H, Montazeri A, Mobini B. The oswestry disability index, the roland-morris disability questionnaire, and the Quebec Back Pain Disability Scale: Translation and validation studies of the Iranian versions. Spine. 2006; 31(14):E454-9. [DOI:10. 1097/01. brs. 0000222141. 61424. f7] [PMID]

[45] Kabat-Zinn J. Full catastrophe living, revised edition: How to cope with stress, pain and illness using mindfulness meditation. New York: Hachette Book Group; 2013.

[46] Praissman S. Mindfulness-based stress reduction: A literature review and clinician's guide. Journal of the American Academy of Nurse Practitioners. 2008; 20(4):212-6. [DOI:10. 1111/j. 1745-7599. 2008. 00306. x] [PMID]

[47] Omidi A, Zargar F. Effect of mindfulness-based stress reduction on pain severity and mindful awareness in patients with tension headache: A randomized controlled clinical trial. Nursing and Midwifery Studies. 2014; 3(3):e21136. [DOI:10. 17795/ nmsjournal21136] [PMID] [PMCID]

[48] Morone NE, Lynch CS, Greco CM, Tindle HA, Weiner DK.I felt like a new person. The effects of mindfulness meditation on older adults with chronic pain: Qualitative narrative analysis of diary entries. The Journal of Pain. 2008; 9(9):841-8. [DOI:10. 1016/j. jpain. 2008. 04. 003] [PMID] [PMCID]

[49] Engels AS, Heller W, Mohanty A, Herrington JD, Banich MT, Webb AG, et al. Specificity of regional brain activity in anxiety types during emotion processing. Psychophysiology. 2007; 44(3):352-63. [DOI:10. 1111/j. 1469-8986. 2007. 00518. x] [PMID]

[50] Williams C. Overcoming depression and low mood: A five areas approach. Boca Raton: CRC Press; 2014. [DOI:10. 1201/b17962]

[51] Parra-Delgado M, LatorrePostigo JM. Effectiveness of mindfulnessbased cognitive therapy in the treatment of fibromyalgia: A randomized trial. Cognitive Therapy and Research. 2013; 37(5):1015-26. [DOI:10. 1007/s10608-013-9538-z]

[52] Greeson JM. Mindfulness research update: 2008. Complementary Health Practice Review. 2009; 14(1):10-8. [DOI:10. 1177/1533210108329862] [PMID] [PMCID]

[53] Ditto B, Eclache M, Goldman N. Short-term autonomic and cardiovascular effects of mindfulness body scan meditation. Annals of Behavioral Medicine. 2006; 32(3):227-34 [DOI:10. 1207/ s15324796abm3203_9] [PMID] 